\title{
Developments in the treatment of moderate to severe ulcerative colitis: focus on adalimumab
}

This article was published in the following Dove Press journal:

Therapeutics and Clinical Risk Management

6 November 2013

Number of times this article has been viewed

\section{Hugh J Freeman}

Department of Medicine (Gastroenterology), University of British Columbia, Vancouver, BC, Canada
Correspondence: Hugh Freeman Gastroenterology, UBC Hospital, 22II Wesbrook Mall, Vancouver, BC, Canada V6T IW5

$\mathrm{Tel}+\mathrm{I} 6048227216$

Fax + I 6048227236

Email hugfree@shaw.ca

\begin{abstract}
In selected patients with moderate to severe active ulcerative colitis who have failed to respond or are poorly responsive to standard pharmacologic forms of treatment with corticosteroids and immunosuppressive agents, therapy with a biological agent may be considered. While infliximab is an established tumor necrosis factor blocker and has a longer history of clinical use, adalimumab is an alternative in the same class and may be employed as an initial biological agent, if indicated for treatment of the disease. Adalimumab may have special appeal to stable users able to self-inject in a home setting rather than a centralized infusion center. Short-term adverse effects have been limited, but long-term adverse events can be serious and remain less well defined. Recently, another agent, subcutaneous golimumab, has also been reported to induce and maintain clinical response and remission in clinical trials, but a large experience has not been accumulated to date in clinical practice. In the future, other biological agents with novel and distinct mechanisms of therapeutic action may become available.
\end{abstract}

Keywords: ulcerative colitis, anti-tumor necrosis factor inhibitors, biological therapy, infliximab, adalimumab

\section{Introduction}

Ulcerative colitis is a chronic mucosal inflammatory disorder of the large intestine. The cause is unknown. Management of patients with idiopathic ulcerative colitis remains highly personalized, in spite of suggested algorithms and practice guidelines that have been published in many excellent literature sources. ${ }^{1-4}$ The present review focuses on adalimumab as an emerging and new form of treatment for inflammatory bowel diseases, and specifically for some patients with ulcerative colitis.

\section{Diagnosis and treatment considerations}

Medical treatment of ulcerative colitis usually follows a precise diagnosis (particularly exclusion of an expanding array of infectious agents that may cause a similar initial clinical illness or be superimposed on ulcerative colitis, particularly infections with cytomegalovirus or Clostridium difficile), an overall clinical evaluation of the patient, that includes consideration of other concomitant medical disorders, recording of drug intolerance and drug hypersensitivities, and evaluation of other critical issues that may influence the treatment decision-making process. Patient age is also an important factor since younger patients, particularly those in the pediatric age group, often have more severe and more extensive disease. Critical in use of many therapeutic agents on an ongoing basis is availability of care, particularly if living in isolated or rural areas with limited tertiary or quaternary levels of expertise, concomitant availability 
of imaging and surgery, patient's occupation, and family support situation, and, especially the costs implicit in different treatment options available in the proposed medical care plan. In some countries, medical care funding may be provided from either government or private sources (or some combination of both), regulatory agencies in different countries may permit approval for specific indications and not others, and with the advent of social media sources of information, specialist physicians can play an important role in accurate interpretation of treatment options and their potential adverse effects. $^{5}$

\section{Treatment goals}

As the cause of ulcerative colitis is not known, there is no known treatment that can cure the disease. However, a number of treatment goals can be envisaged that lead to induction of clinical remission and improvement in quality of life. To some extent, this depends on initial evaluation of the clinical severity of disease, confirmation of the diagnosis, usually involving endoscopic and histologic examinations, and, at present, evaluation of the extent of disease. ${ }^{5}$ Imaging of the disease is best done early in the course of the clinical evaluation, prior to initiation of treatment, and subsequently, if a change in management is contemplated. High quality photodocumentation of the entire colon due to the rapid evolution of endoscopic technology and the emergence of higher definition colonoscopes with digital imaging is now available. Serial imaging files defining the macroscopic appearance of the disease in individuals can now be compared over time. These have been used for "real-life" clinical evaluation as well as clinical trials to assess different therapeutic agents. Documentation of endoscopic mucosal appearance may be useful, but is also burdened by interobserver variation in the observation and description of macroscopic mucosal changes, including ulcerative colitis. To some degree, this variability may be limited with added experience of individual endoscopists, but even if improvement is detected, only partial, rather than complete healing has occurred. Histologic evidence of mucosal healing throughout the colon would appear to be a more objective parameter but at present this is not usually an approach recommended in therapeutic trials. Correlation between the global clinical status of the patient, macroscopic changes defined by endoscopists, and microscopic changes observed by experienced histopathologists has limitations.

\section{Mucosal healing}

Assessment of mucosal healing in inflammatory bowel disease has been evaluated previously and reviewed elsewhere, ${ }^{6}$ and remains as a marker in clinical trials of new agents as well as in routine clinical practice. A number of endoscopic scoring methods have been developed, especially for use in clinical trials, and reviews of different clinical, endoscopic, and histologic endpoints used in inflammatory bowel disease, particularly in ulcerative colitis, have been published and have emphasized the absence of any standardized definition. ${ }^{7-10}$ Long-term studies have suggested that evaluation of the effects of specific treatment regimens on endoscopic changes, as well as the clinical response to treatment, may be important. It is believed that the prognosis in an individual patient may be better if "mucosal healing" can be achieved. ${ }^{10}$ More recent clinical trials of different therapeutic agents often include an evaluation of mucosal healing because of the possible influence on duration of remission, disease complications, particularly colorectal cancer, need for colectomy, and quality of life. Mucosal healing per se, however, is really an assessment of the macroscopic features of the disease alone, rather than histologic healing. It may or may not represent a true advancement in treatment evaluation, even in clinical trials, although endoscopic evidence of mucosal healing after a 1 -year period has been reported to result in a reduced need for active treatment and a reduced risk for colectomy in ulcerative colitis. ${ }^{11}$ However, most involved in evaluation of patients with ulcerative colitis would recognize that endoscopic "mucosal healing" and resolution of the inflammatory process as reflected in histologic remission are not equivalent ${ }^{10}$ and, at this stage, more conceptual definitions (eg, so-called “deep remission") to evaluate and monitor treatment outcomes appear to be emerging. ${ }^{12,13}$

\section{Disease severity}

Patients may be classified on the basis of the clinical severity of the colonic inflammatory disease process along with the extent of disease. ${ }^{5}$ Clinical severity in ulcerative colitis has traditionally been dependent upon a few clinical endpoints. The disease has been defined as mild if there are less than four bowel motions per day and moderate if there are more than four bowel motions per day with limited systemic toxicity (pulse more than 90 per minute, temperature less than $37.5^{\circ} \mathrm{C}$, hemoglobin greater than $10.5 \mathrm{~g} / \mathrm{dL}$, or erythrocyte sedimentation rate less than $30 \mathrm{~mm}$ per hour). Severe ulcerative colitis was defined by Truelove and Witts ${ }^{14}$ largely on the basis of clinical criteria, specifically more than six bloody bowel motions per day with one or more signs of systemic toxicity. Each of these markers are assumed to reflect the colonic inflammatory process, but other concomitant chronic diseases may also be significant (eg, ankylosing spondylitis). 
C-reactive protein and other markers, such as fecal calprotectin, have also been used by some as more "objective" markers of the severity of the inflammatory process. In general, gauging the clinical severity of the inflammatory process may define the therapeutic approach. Traditionally, mild to moderate disease may be treated initially with a 5-aminosalicylate-containing medication alone, while moderate to severe disease may lead to earlier treatment with other measures, including corticosteroids with or without an immunosuppressive agent (eg, azathioprine), to control the inflammatory process. For corticosteroids, systemic formulations or more site-specific agents, like budesonide with a specific carrier (ie, budesonide-MMX extended release tablets; Santarus, Inc., San Diego, CA, USA), have been used. ${ }^{15}$ The extent of the disease may also be evaluated using modern imaging methods, including endoscopic modalities, that may determine the extent of mucosal involvement as localized rectal disease, or proctitis, distal or left-sided disease, and more extensive ulcerative colitis, usually extending in a continuous pattern proximal to the splenic flexure. In some with extensive disease, pancolonic involvement occurs; however, urgent and extended pancolonoscopy is rarely necessary to evaluate the extent of disease in severely ill patients because there are increased risks to endoscopic evaluation in this setting.

\section{Extent of disease}

To some degree, the clinical severity often mirrors the extent of disease involvement. Acute severe disease may also occur, often with the initial clinical event, and may be referred to as severe or toxic colitis (as opposed to toxic megacolon, a disorder that generally requires urgent colectomy rather than medical treatment). If the extent of involvement is limited, then only a local topical inflammatory agent may be needed, rather than orally administered forms of medication. Practically, in those with proctitis, some with this limited form of inflammatory disease may still prefer oral treatment over rectal administration. For most patients with moderate to severe inflammatory disease, the process is far more extensive in the colon and consideration of other agents to control the inflammatory process may be necessary, including biological agents, such as adalimumab.

\section{Use of biological agents in ulcerative colitis}

Studies on the use of tumor necrosis factor (TNF) blockers in Crohn's disease have previously been extensively examined and reviewed. ${ }^{16}$ In patients with moderately active to severely active ulcerative colitis, most specialist physicians will resort to management with conventional treatment regimens using corticosteroids and/or immunosuppressive drugs, including azathioprine or 6-mercaptopurine. However, in some of these patients, treatment failure occurs, as reflected in a poor response to conventional pharmacologic therapies or a failure to achieve remission, usually defined by clinical and/or endoscopic measurements. For these difficult-tomanage patients, added biological agents have been useful for some, providing an additional management approach. Infliximab was first approved by the US Food and Drug Administration in 2006 for use if an inadequate response to conventional treatment of moderately to severely active disease was documented. This agent is an intravenously administered monoclonal antibody against TNF-alpha. In the so-called ACT (Acute ulcerative Colitis Treatment) 1 and ACT 2 clinical trials with two different doses of infliximab, safety and efficacy were shown over a non-infliximab placebo group. ${ }^{17}$ Later, in 2012, a second monoclonal agent that could be subcutaneously administered, adalimumab, also received formal Food and Drug Administration approval. To date, none of these agents have been evaluated in head-to-head clinical trials, but a recent cost-per-remission analysis for adults with moderate to severe ulcerative colitis suggested a lower cost for infliximab compared with adalimumab. ${ }^{18}$ Additional cost evaluations are needed. Recently, subcutaneous golimumab, another human monoclonal antibody to TNF-alpha, was initially reported in clinical trials to induce a clinical response and remission and later maintain a clinical response in moderate to severe ulcerative colitis. ${ }^{19,20}$

\section{Clinical efficacy and safety studies with adalimumab}

Some early open-label studies and case reports described a possible role for adalimumab as a second biological agent for use in treatment of some patients with difficult-to-manage ulcerative colitis. These initial reports resulted in subsequent multicenter and randomized clinical trials on adalimumab for treatment of moderate to severe ulcerative colitis, termed ULTRA (Ulcerative Colitis Long-Term Remission and Maintenance with Adalimumab Treatment of Moderate to Severe Ulcerative colitis) 1 and ULTRA 2. ${ }^{21,22}$ ULTRA 1 was an 8-week, double-blind, placebo-controlled trial designed to assess induction of clinical remission in patients with moderate to severe ulcerative colitis who had not previously been treated with an anti-TNF-alpha agent. Over an 8-week period, adalimumab was significantly better than a "matching" placebo group (ie, no other details published 
on placebo per se). ${ }^{21}$ ULTRA 2 was designed as a 52-week clinical trial to gather longer-term data for adalimumab in the treatment of moderate to severe ulcerative colitis. Use of corticosteroids and immunomodulators were permitted in both the adalimumab and placebo groups, at least early in the clinical trial, and approximately $40 \%$ of the patient population evaluated had been exposed to prior infliximab. ${ }^{22}$ Over the course of the ULTRA 2 evaluation, adalimumab was considered to be more effective than the placebo treatment for induction and maintenance of clinical remission in moderate to severe ulcerative colitis, while mucosal healing using endoscopic assessment was also noted to be significant over placebo through to 1 year. ${ }^{22}$

Subsequent studies evaluated longer-term outcomes of specific patient subgroups thought to have responded to induction therapy with adalimumab and later analyzed from the ULTRA 2 evaluation. For example, analysis of an "early responder" subgroup suggested that these patients were more likely to have a positive clinical outcome at 1 year. ${ }^{23}$ In contrast, an independent evaluation of a separate group of adalimumab-treated patients suggested that only a limited proportion of patients remain clinically well on continued treatment at 2 years. ${ }^{24}$

\section{Use in other clinical settings}

Treatment of other colitis-associated issues has also been addressed in some clinical studies since the therapeutic role of biological agents may not simply relate to ulcerative colitis (or Crohn's disease) per se. For example, the appearance of pyoderma gangrenosum, an extraintestinal dermatologic manifestation of inflammatory bowel disease, ${ }^{25}$ often requires aggressive management of the underlying inflammatory bowel disorder, usually with corticosteroids as first-line therapy. In some patients, however, satisfactory healing of this skin disorder does not appear to occur. In some of these, biologic therapies, either with infliximab or adalimumab, may be considered. ${ }^{26}$

Chronic pouchitis frequently develops in patients with ulcerative colitis after post-colectomy reconstructive surgery. In some, post-colectomy symptoms associated with chronic pouchitis may be severe. In an open-label series of patients with previously treated pouchitis, clinical remission and avoidance of permanent ileostomy was reported with adalimumab, including patients with prior failed infliximab treatment. ${ }^{27}$

\section{Mechanisms of action of adalimumab}

Molecular, cellular, physiologic, and biological properties of TNF-alpha antibodies have been recently and extensively reviewed for Crohn's disease, but this information is also directly relevant for patients with ulcerative colitis being considered for TNF-alpha blocker therapy. ${ }^{16}$ A great deal of information has been forthcoming in the recent past as this continues to be an area of intense investigative activity. TNF is a cytokine that initiates a defensive response to local tissue injury. Innate and adaptive immune responses cause an increase in serum and mucosal TNF, ultimately influencing the chronicity of the inflammatory response. TNF is a membrane protein (tmTNF) that forms a soluble protein (sTNF) due to the proteolytic action of a TNF-alpha protease-converting enzyme. Cellular responses to stimuli such as bacterial lipopolysaccharide may result from reverse signaling by tmTNF. The sTNF and precursor tmTNF bind to two different TNF receptors (TNFR1 and TNFR2), leading to expression of interleukin-1, interleukin-6, interferongamma, and other cell adhesion molecules, as well as some inflammatory molecular mediators. TNFR1 is considered to be responsible for the proinflammatory properties of TNF, while TNFR2 plays an immunoregulatory role. Activation of apoptosis may result, and depending on host genetic makeup, timing of TNF release and local TNF concentrations, immunostimulatory or immunosuppressive effects can occur. Both infliximab and adalimumab may induce apoptosis in peripheral blood monocytes as well as intestinal mucosal T-cells. Interestingly, certolizumab, an agent also studied in Crohn's disease, does not appear to produce apoptosis via tmTNF, possibly because of an inability to form crosslinkages with tmTNF. This may suggest that mechanisms other than apoptosis per se may be important in the clinical effects of TNF-alpha agents. Adalimumab antibodies may develop during use, resulting in reduced serum adalimumab concentrations and effectiveness, possibly by formation of large multivalent immune complexes with TNF-alpha antibodies resulting in rapid clearance from the circulation.

\section{Risks and management issues with adalimumab}

A list of potential adverse effects of anti-TNF-alpha agents has accumulated in patients treated in open-label and controlled clinical trials as well as in post-marketing evaluations and clinical use in patients with inflammatory bowel disease. ${ }^{16}$ A complete and extensive listing of these adverse effects may also be found in any standard desk reference, and potential users of any anti-TNF-alpha agent should be made aware of each of these by the prescribing physician. Even if an adverse event has not been noted specifically for adalimumab, adverse events with any monoclonal TNF-alpha 
antibody should be noted. These have included cardiovascular events, particularly severe cardiac failure; hematologic events including aplastic anemia with pancytopenia or deficiency of any single hematocytologic component; hypersensitivity reactions including rash, anaphylactoid reaction, urticaria, and drug eruptions; a spectrum of autoimmune reactions ranging from the subclinical appearance of autoantibodies to a rare, lupus-like syndrome; neurologic events including new onset or exacerbation of clinical symptoms and radiologic evidence of demyelinating disease, including multiple sclerosis; and immune suppressive effects resulting in infections, particularly with opportunistic or other ubiquitous agents as well as malignancies, including lymphoma. Others were unable to define a specific association between specific, but reported, new-onset neurologic adverse events and TNFalpha blockers. ${ }^{28}$ Interestingly, however, adalimumab has also been noted to precipitate onset of inflammatory bowel disease, specifically ulcerative colitis, in a case of a patient with already established arthritis. ${ }^{29}$

A thorough clinical assessment must be done prior to initiation of any anti-TNF-alpha agent. Absolute and relative contraindications for use of anti-TNF agents, including adalimumab, have been previously noted elsewhere. ${ }^{16}$ These include suspicion of an underlying septic focus, specifically, an abscess, exacerbation of ulcerative colitis due to an enteric infection (eg, $C$. difficile, cytomegalovirus), severe congestive heart failure, uncontrolled human immunodeficiency virus disease, endemic mycosis, evidence of tuberculosis (positive interferon gamma assay and/or chest X-ray prior to a 4-week course of isoniazid), and suspected multiple sclerosis. Caution in use has also been recommended for a past history of other demyelinating neurologic disorders (eg, Guillain-Barré syndrome, optic neuritis), chronic liver disease, and malignancies (particularly lymphoma and leukemia). Finally, known pregnancy or planned pregnancy should be considered. Both infliximab and adalimumab have been classified by the Food and Drug Administration as pregnancy class B agents, so use may be considered during the first two trimesters if the agents are thought to be absolutely necessary. A recent authoritative review indicated that antiTNF agents can cross the placenta in the latter part of the second trimester of gestation, although they appear to be safe, at least in the short-term. ${ }^{30}$ Small amounts of anti-TNF may be transferred in breast milk, however, so a deleterious effect of this exposure to breast milk on the newborn, although unlikely, cannot be excluded. ${ }^{30}$

Vaccination status of the patient with ulcerative colitis should be immediately reviewed and updated prior to use of
TNF-alpha antibodies because patients with inflammatory bowel disease are at risk for vaccine-preventable illnesses. ${ }^{31}$ Some guidelines for immunizations in inflammatory bowel disease have previously appeared. ${ }^{32}$ Live vaccines should be avoided, specifically measles-mumps-varicella, varicella (oral), yellow fever vaccine, and oral polio vaccine. If the patient is hepatitis B virus-positive, regardless of hepatitis B surface antigen status, treatment for hepatitis B should be initiated before administration of an anti-TNF-alpha agent, including adalimumab.

Travel issues are also important in candidates for biological agents. For travel, regular precautions should be taken for malaria. Ciprofloxacin and/or metronidazole should be available for use in the event of onset of traveler's diarrhea. Medical records that include specific medications and dosages of all prescribed for ulcerative colitis should be documented, and consultation records, travel insurance, and contact names of specialist physicians in foreign countries with experience in management of colitis are useful.

\section{Summary}

Overall, in selected patients with moderate to severe active ulcerative colitis who have failed completely or are poorly responsive to standard pharmacologic forms of treatment with corticosteroids and immunosuppressive agents, treatment with a biological agent should be considered given that randomized clinical trials have demonstrated some effectiveness in the short-term over placebo, although not over each other. While infliximab has a longer history of clinical use, adalimumab may well be employed as the initial biological agent in this setting, if a biological agent is indicated. In particular, adalimumab may have special appeal to experienced, particularly stable users who are able to self-inject in a home setting rather than requiring a centralized infusion center. Although short-term adverse events have been limited in those with no specific contraindication to their use, long-term adverse events can be serious but are less well known. Most of these to date largely relate to immunosuppression induced by this class of biological agents. In the near future, other agents with a different mechanism of therapeutic action, such as vedolizumab, ${ }^{33}$ may further the clinical experience with these biological agents, including patients with difficult-to-treat moderate to severe ulcerative colitis. Additional studies will be needed to explore the relative advantages and disadvantages of new and emerging "next generation monoclonal antibodies"34 with possible biosimilar therapeutic properties that promise to pose difficult regulatory challenges. 


\section{Disclosure}

The author reports no conflict of interest in this work.

\section{References}

1. Mowat C, Cole A, Windsor A, et al; IBD Section of the British Society of Gastroenterology. Guidelines for the management of inflammatory bowel disease in adults. Gut. 2011;60:571-607.

2. Meier J, Sturm A. Current treatment of ulcerative colitis. World $J$ Gastroenterol. 2011;17:3204-3212.

3. Ordas I, Eckmann L, Talamini M, Baumgart DC, Sandborn WJ. Ulcerative colitis. Lancet. 2012;380:1609-1619.

4. Armuzzi A, Pugliese D, Nardone OM, Guidi L. Management of difficultto-treat patients with ulcerative colitis: focus on adalimumab. Drug Des Devel Ther. 2013;7:289-296.

5. IBD and ulcerative colitis. In: Bayless TM, Hanauer SB, editors. Advanced Therapy in Inflammatory Bowel Disease. Shelton, CT: People's Medical Publishing House; 2011;1-621.

6. Freeman HJ. Limitations in assessment of mucosal healing in inflammatory bowel disease. World J Gastroenterol. 2010;16:15-20.

7. D'Haens G, Sandborn WJ, Feagan BG, et al. A review of activity indices and efficacy end points for clinical trials of medical therapy in adults with ulcerative colitis. Gastroenterology. 2007;132:763-786.

8. Kornbluth A, Sachar DB. Ulcerative colitis practice guidelines in adults (update): American College of Gastroenterology, Practice Parameters Committee. Am J Gastroenterol. 2004;99:1371-1385.

9. Geboes K, Dalle I. Influence of treatment on morphological features of mucosal inflammation. Gut. 2002;50 Suppl 3:III37-III42.

10. Villanacci V, Antonelli E, Geboes K, Casella G, Bassotti G. Histological healing in inflammatory bowel disease: a still unfulfilled promise. World J Gastroenterol. 2013;19:968-978.

11. Frøslie KF, Jahnsen J, Moum BA, Vatn MH; IBSEN Group. Mucosal healing in inflammatory bowel disease: results from a Norwegian population-based cohort. Gastroenterology. 2007;133:412-422.

12. Zallot C, Peyrin-Biroulet L. Deep remission in inflammatory bowel disease: looking beyond symptoms. Curr Gastroenterol Rep. 2013;15:315.

13. Colombel JF, Louis E, Peyrin-Biroulet L, Sandborn WJ, Panaccione R. Deep remission: a new concept? Dig Dis. 2012;30 Suppl 3:107-111.

14. Truelove SC, Witts CJ. Cortisone in ulcerative colitis. Final report on a therapeutic trial. Br Med J 1955;ii:1041-1048.

15. Sandborn WJ, Travis S, Moro L, et al. Once-daily budesonide MMX extended-release tablets induce remission in patients with mild to moderate ulcerative colitis: results from the CORE 1study. Gastroenterology. 2012;143:1218-1226.

16. Thomson AB, Gupta M, Freeman HJ. Use of the tumor necrosis factor-blockers for Crohn's disease. World J Gastroenterol. 2012;18: 4823-4854.

17. Rutgeerts P, Sandborn WJ, Feagan BG, et al. Infliximab for induction and maintenance therapy for ulcerative colitis. NEngl J Med. 2005;353: 2462-2476.

18. Lofland JH, Mallow P, Rizzo J. Cost-per-remission analysis of infliximab compared to adalimumab among adults with moderate-to-severe colitis. J Med Econ. 2013;16:461-467.

Therapeutics and Clinical Risk Management

\section{Publish your work in this journal}

Therapeutics and Clinical Risk Management is an international, peerreviewed journal of clinical therapeutics and risk management, focusing on concise rapid reporting of clinical studies in all therapeutic areas, outcomes, safety, and programs for the effective, safe, and sustained use of medicines. This journal is indexed on PubMed Central, CAS,
19. Sandborn WJ, Feagan BG, Marano C, et al. Subcutaneous golimumab induces clinical response and remission in patients with moderate to severe ulcerative colitis. Gastroenterology. June 2, 2013. [Epub ahead of print.]

20. Sandborn WJ, Feagan BG, Marano C, et al. Subcutaneous golimumab maintains clinical response in patients with moderate-to-severe ulcerative colitis. Gastroenterology. June 13, 2013. [Epub ahead of print.]

21. Reinisch W, Sandborn WJ, Hommes DW, et al. Adalimumab for induction of clinical remission in moderately to severely active ulcerative colitis: results of a randomized controlled trial. Gut. 2011;60: 780-787.

22. Sandborn WJ, van Assche G, Reinisch W, et al. Adalimumab induces and maintains clinical remission in patients with moderate-to-severe ulcerative colitis. Gastroenterology. 2012;142:257-265.

23. Sandborn WJ, Colombel JF, D'Haens G, et al. One-year maintenance outcomes among patients with moderately-to-severely active ulcerative colitis who responded to induction therapy with adalimumab: subgroup analyses from ULTRA 2. Aliment Pharmacol Ther. 2013;37:204-213.

24. McDermott E, Murphy S, Keegan D, O’Donoghue D, Mulcahy H, Doherty G. Efficacy of adalimumab as a long term maintenance therapy in ulcerative colitis. J Crohns Colitis. 2013;7:150-153.

25. Freeman HJ. Erythema nodosum and pyoderma gangrenosum in 50 patients with Crohn's disease. Can J Gastroenterol. 2005;19: 603-606.

26. Arguelles-Arias F, Castro-Laria L, Lobaton T, et al. Characteristics and treatment of pyoderma gangrenosum in inflammatory bowel disease. Dig Dis Sci. 2013;58:2949-2954.

27. Barreiro-de Acosta M, Garcia-Bosch O, Gordillo J, et al. Efficacy of adalimumab rescue therapy in patients with chronic refractory pouchitis previously treated with infliximab: a case series. Eur J Gastroenterol Hepatol. 2012;24:756-758.

28. Deepak P, Stobaugh DJ, Sherid M, Sifuentes H, Ehrenpreis ED. Neurological events with tumor necrosis factor alpha inhibitors reported to the Food and Drug Administration Adverse Event Reporting System. Aliment Pharmacol Ther. 2013;38:388-396.

29. Tursi A, Penna A. Onset of ulcerative colitis under treatment with adalimumab. Am J Gastroenterol. 2008;103:2410-2412.

30. Gisbert JP, Chaparro M. Safety of anti-TNF agents during pregnancy and breastfeeding in women with inflammatory bowel disease. Am J Gastroenterol. 2013;108:1426-1438.

31. Melmed GY, Ippoliti AF, Papdakis KA, et al. Patients with inflammatory bowel disease are at risk for vaccine-preventable illnesses. Am J Gastroenterol. 2006;101:1834-1840.

32. Sands BE, Cuffari C, Katz J, et al. Guidelines for immunizations in patients with inflammatory bowel disease. Inflamm Bowel Dis. 2004;10: 677-692.

33. Feagan BG, Rutgeerts P, Sands BE, et al. Vedolizumab as induction and maintenance therapy for ulcerative colitis. $N$ Engl J Med. 2013;369: 699-710.

34. Declerck PJ. Biosimilar monoclonal antibodies: a science-based regulatory challenge. Exp Opin Biol Ther. 2013;13:153-156.
EMBase, Scopus and the Elsevier Bibliographic databases. The manuscript management system is completely online and includes a very quick and fair peer-review system, which is all easy to use. Visit http://www.dovepress.com/testimonials.php to read real quotes from published authors. 